\title{
ROBUST SECOND ORDER SLIDING MODE CONTROLLER FOR ELECTROPNEUMATIC ACTUATOR
}

\author{
S. Laghrouche ${ }^{\dagger}$, M. Smaoui ${ }^{\ddagger}$, X. Brun ${ }^{\ddagger}$ and F. Plestan ${ }^{\dagger *}$ \\ ${ }^{\dagger}$ IRCCyN, Ecole Centrale de Nantes / CNRS BP 92101, 1 Rue de la Noë, 44321 Nantes Cedex 03, France, \\ \{Salah. Laghrouche, Franck.Plestan\}@irccyn.ec-nantes.fr,www.irccyn.ec-nantes.fr \\ ${ }^{\ddagger}$ LAI - INSA de Lyon, Bât. Saint-Exupery, 25, avenue Jean Capelle, 69621 Villeurbanne cedex, France, \\ \{smaoui, xavier.brun\}elai.insa-lyon.fr, www-lai.insa-lyon.fr
}

Keywords: Second order sliding mode, electropneumatic actuator, robust controller, experimental set-up.

\begin{abstract}
This paper deals with the robust control problem of a pneumatic actuator subjected to parameters uncertainties and load disturbances. The control strategies are based on second order sliding mode approaches. Implementation results of the proposed sliding mode control schemes on an experimental set-up are given to illustrate the developments.
\end{abstract}

\section{Nomenclature}

$y \quad$ Load position $(m)$

$v$ Load velocity $(\mathrm{m} / \mathrm{s})$

$u \quad$ Servo-distributor input voltage $(V)$

$p$ Pressure $(\mathrm{Pa}) \quad V$ Chamber volume $\left(\mathrm{m}^{3}\right)$

$F_{\text {ext }}$ External force $(N) \quad F_{f}$ Dry friction forces $(N)$

$b$ Viscous friction coeff. $\quad M$ Load mass $(\mathrm{kg})$

$k$ Polytropic constant $T$ Chamber temperature $(K)$

$r \quad$ Perfect gas constant related to unit mass $(\mathrm{J} / \mathrm{kg} / \mathrm{K})$

$S \quad$ Area of the cylinder piston on a chamber side $\left(\mathrm{m}^{2}\right)$

$q_{m} \quad$ Mass flow rate provided by the servo-distributor to cylinder chamber $(\mathrm{kg} / \mathrm{s})$

$N \quad$ Relative to $N$ chamber

$P \quad$ Relative to $P$ chamber.

\section{Introduction}

Until several years, pneumatic actuators were mainly used for two-end position control. However, viewed that the pneumatic actuators have several advantages versus for example electrical or hydraulic ones because they are cheap, lightweight, clean, and they present a good force/weight ratio, many works and developements showed that this kind of actuators can also be used in a position control context, by using specific servodistributor $[9,31,10,14,16,21,27]$. As the necessary technology is avalaible for the positioning control of pneumatic actuators, the exciting challenge is now to develop new control laws able to get high static and dynamic precision in presence of undesirable characteristics. They are due to the high compressibility of

\footnotetext{
${ }^{*}$ Corresponding author
}

air, the high other nonlinearities of these systems (in particular frictions), and the variation of environment as load disturbance. Due to these latter facts, several works have proposed linear and nonlinear controllers in order to get high performances behaviour. In [7], a comparison between two positionning linear control laws (a fixed gains control law and a control law with scheduling gains) of an electropneumatic disymmetrical cylinder is made in point to point displacement aim. This work has been extended to nonlinear control in [23, 6] and [8], in which a linearizing controller has been implemented on an experimental set-up in single and multi variable context. Due to uncertainties appearing in the modelization, robust controllers are necessary to ensure position tracking with high precision. A possible way to reach this objective is the use of sliding mode approach. This approach has been used in several works $[5,22,30]$. The standard sliding mode features are high accuracy and robustness with respect to various internal and external disturbances. Specific problem involved by this technique is chattering effect, i.e. dangerous high-frequency vibrations of the controlled system. Some authors [26, 29] relate the chattering behaviour to the discontinuity of the discontinuous "sign" function on the sliding manifold. To overcome this problem, the first and natural suggestion is to replace the discontinuous function in a small vicinity of the surface by a smooth approximation; that implies a small deterioration in accuracy and robustness ${ }^{1}$. Recently, a new philosophy for sliding called "high-order sliding mode" has been proposed with several different solutions (twisting and super-twisting algorithms [19], approach based on bang-bang control [1], $L Q$ approach for the definition of the sliding manifold [18]). Instead of influencing the first sliding manifold derivative, the "sign" function acts on its higher time derivative. Keeping the main advantages of the standard sliding mode control, the chattering effect is eliminated and higher order precision is provided. It has been shown in [17] for the control of a permanent magnet synchronous motor.

The paper proposes a second order sliding mode controller for an electropneumatic actuator. Section 2 describes the model of the electropneumatic actuator and states the problem under

\footnotetext{
${ }^{1}$ Note that this solution is not enough in pneumatic field [4]: indeed, a good compromise between static position error and chattering cannot be found. So, the spool of the valve is exited which conduced to a lot of noise due to the air going from source to exhaust and an undesirable deterioration of the servodistributor
} 
interest. Section 3 deals with the design of a second order sliding mode controller for this system. Section 4 discusses the implementation results of the proposed control schemes on an experimental set-up.

\section{Model of the pneumatic system and control problem statement}

\subsection{Description of the experimental set-up}

The electropneumatic system under interest is a double acting actuator (Figure 1) composed by two chambers, denoted $P$ (as positive) and $N$ (as negative). The air mass flow rates entering the two chambers are modulated by two three-way servodistributors controlled by a micro-controller with two electrical inputs of opposite signs. The pneumatic jack horizontally moves a load carriage of mass $\mathrm{M}$, has a stroke of $500 \mathrm{~mm}$ and is very unsymmetrical since it has an internal diameter of $32 \mathrm{~mm}$ with a simple rod of $20 \mathrm{~mm}$ diameter. The position sensor of the load cariage is a potentiometer. Velocity is obtained by analog derivation from the position signal and a numerical derivation of the velocity signal gives the acceleration information used by the control law. Two pressure sensors are also implemented in each chamber and used for incremented the quality of control in term of accuracy and robustness.

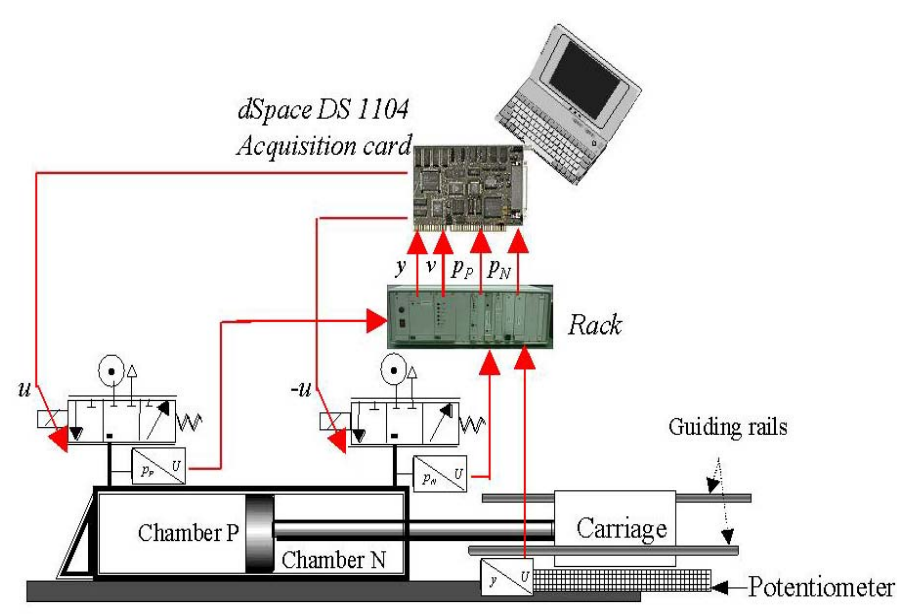

Figure 1: Electropneumatic system

\subsection{Model}

Assumptions $[25,20]$ used to obtain a model of the pneumatic part of the electropneumatic system are:

- The supply and exhaust pressures are constant,

- The air is a perfect gas and its kinetic energy is negligible in both chambers,

- The pressure and the temperature are homogeneous in each chamber,
- The thermodynamic evolution of the air in the cylinder chambers is polytropic and characterized by a coefficient $k$,

- The temperature variations in each chamber are negligible with regards to the mean temperature $\mathrm{T}$,

- There is no mass flow leakage between the two cylinder chambers and outside the actuator,

- The dynamics of the servo-distributor are neglected.

- The two three-way servodistributors are the same and their electrical variable inputs are of inverse signs.

Then, a nonlinear dynamic model of the electropneumatic system reads as:

$$
\begin{aligned}
\dot{p}_{P} & =\frac{k r T}{V_{P}(y)}\left[q_{m}\left(u, p_{p}\right)-\frac{S_{P}}{r T} p_{P} v\right] \\
\dot{p}_{N} & =\frac{k r T}{V_{N}(y)}\left[q_{m}\left(-u, p_{N}\right)+\frac{S_{N}}{r T} p_{N} v\right] \\
\dot{v} & =\frac{1}{M}\left[S_{P} p_{P}-S_{N} p_{N}-b v-F_{f}-F_{e x t}\right] \\
\dot{y} & =v
\end{aligned}
$$

with $y$ the load carriage position, $v$ its velocity and $p_{P}$ and $p_{N}$ the pressures of $P$ and $N$ chambers. The model of mass flow rate delivered by each servo-distributor can be reduced to a static function described by two relationships $q_{m}\left(u, p_{P}\right)$ and $q_{m}\left(-u, p_{N}\right)$. The two first equations of (1) concern the pneumatic part of the system and are obtained from the state equation of perfect gases, the mass conservation law and the polytropic law under the assumptions given above. The two last equations describe the mechanical part and are derived from the fundamental mechanical equation applied to the moving part. The term $F_{f}$ represents all the dry friction forces which act on the moving part in presence of viscous friction $(b . v)$ and an external force only due to atmospheric pressure $\left(F_{\text {ext }}\right)$. In order to get an affine nonlinear state model, the mass flow rate static characteristic issued from measurements [24] is written as a function of control input $u$ and polynomial functions of $p_{P}$ and $p_{N}$ [3]

$$
\begin{aligned}
& q_{m}\left(u, p_{P}\right)=\varphi\left(p_{P}\right)+\psi\left(p_{P}, \operatorname{sgn}(u)\right) \cdot u \\
& q_{m}\left(-u, p_{N}\right)=\varphi\left(p_{N}\right)-\psi\left(p_{N}, \operatorname{sgn}(-u)\right) \cdot u
\end{aligned}
$$

\subsection{Uncertainties}

Two kinds of uncertainties are taken into account: uncertainties due to the identification of physical parameters, and variations of environment.

\begin{tabular}{|l||c|}
\hline Viscous friction coefficient & $\pm 20 \%$ \\
\hline Dry friction coefficient & $\pm 90 \%$ \\
\hline Function $\varphi(\cdot)$ & $\pm 15 \%$ \\
\hline Function $\psi(\cdot, \cdot)$ & $\pm 5 \%$ \\
\hline Load mass variation & $\pm 50 \%$ \\
\hline
\end{tabular}


The knowledge of the viscous friction coefficient has been identified and the variation of this coefficient around the nominal value has been experimentally evaluated at $\pm 20 \%$. The dry friction coefficient is more difficult to identify: the track surface quality (thus the piston position), the seal wear, the working conditions (temperature, pressure, quality of air) influence the dry friction values. By some experimental tests, dry friction variation around the nominal value is evaluated to $\pm 90 \%$. Futhermore, the dry friction variations are supposed to be not instantaneous: the dry friction dynamics are then bounded. The mass flow rate delivered by each servodistributor has been approximated by polynomial functions (2). The uncertainties on $\varphi(\cdot)$ and $\psi(\cdot)$ are evaluated to $\pm 15 \%$ and $\pm 5 \%$ respectively. Finally, during the load moving, the total mass in displacement can evolve from $17 \mathrm{~kg}$ until $47 \mathrm{~kg}$. The nominal mass is $32 \mathrm{~kg}$, the variation being more and less $\pm 50 \%$.

\subsection{State model with uncertainties}

The formalization of the variations is stated as

$$
\begin{array}{lll}
k r T \varphi\left(p_{P}, u\right) & =k_{1}=k_{01}+\delta k_{1}, \\
k r T \psi\left(p_{P}, \operatorname{sign}(u)\right) & =k_{2}=k_{02}+\delta k_{2}, \\
-k S_{p} & =k_{3}=k_{03}+\delta k_{3}, \\
k r T \varphi\left(p_{N}, u\right) & =k_{4}=k_{04}+\delta k_{4}, \\
-k r T \psi\left(p_{N}, \operatorname{sign}(-u)\right) & =k_{5}=k_{05}+\delta k_{5}, \\
k S_{N} & =k_{6}=k_{06}+\delta k_{6}, \\
S_{P} / M & =k_{7}=k_{07}+\delta k_{7}, \\
-S_{N} / M & =k_{8}=k_{08}+\delta k_{8}, \\
-b / M & =k_{9}=k_{09}+\delta k_{9} .
\end{array}
$$

where $k_{0 i}(1 \leq i \leq 9)$ is the nominal value of the concerned parameter, $\delta k_{i}$ the uncertainty on the concerned parameter such that $\left|\delta k_{i}\right| \leq \delta k_{0 i}$, with $\delta k_{0 i}$ a known positive bound. Note that, viewed the previous hypotheses, $\delta k_{3}=\delta k_{6}=0$. The term $F=-\frac{F_{f}+F_{e x t}}{M}$ is viewed as a perturbation which is bounded, as its first time derivative. Let $x$ denote the state

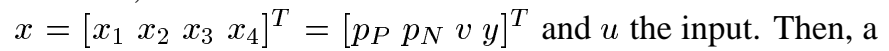
state space model of the pneumatic actuator is

$$
\begin{aligned}
& \dot{x}_{1}=\frac{1}{V_{P}\left(x_{4}\right)}\left[k_{1}+k_{2} \cdot u+k_{3} \cdot x_{1} \cdot x_{3}\right] \\
& \dot{x}_{2}=\frac{1}{V_{N}\left(x_{4}\right)}\left[k_{4}+k_{5} \cdot u+k_{6} \cdot x_{2} \cdot x_{3}\right] \\
& \dot{x}_{3}=k_{7} \cdot x_{1}+k_{8} \cdot x_{2}+k_{9} \cdot x_{3}+F \\
& \dot{x}_{4}=x_{3}
\end{aligned}
$$

with $x \in \mathcal{X} \subset \mathbb{R}^{4}$ and $u \in \mathcal{U} \subset \mathbb{R}$ such that $\mathcal{X}=\{x \in$ $\mathbb{R}^{4}\left|, 0<x_{\text {min }} \leq x_{i} \leq x_{\text {MAX }}, 1 \leq i \leq 2, x_{\text {imin }} \leq\right| x_{i} \mid \leq$ $\left.x_{i M A X}, 3 \leq i \leq 4\right\}$ and $\mathcal{U}=\left\{u \in \mathbb{R}|| u \mid \leq u_{M A X}\right\}$, $x_{\text {min }}$ and $x_{\text {MAX }}$ the minimum/maximum values of the $P$ and $N$ chambers pressures, $x_{3 \min }$ and $x_{3 M A X}$ (resp. $x_{4 \min }$ and $\left.x_{4 M A X}\right)$ the minimum/maximum values of the load velocity (resp. position) and $u_{M A X}$ the maximum value of the voltage input.

\subsection{Control problem statement}

The aim of the control law is to respect a good accuracy in term of position tracking for a desired trajectory defined by a fifth order time polynomial function (Figure 2). The amplitude of displacement is equal to $50 \%$ of the total stroke around the central position and the maximum desired velocity equals 0.60 $\mathrm{m} / \mathrm{s}$.

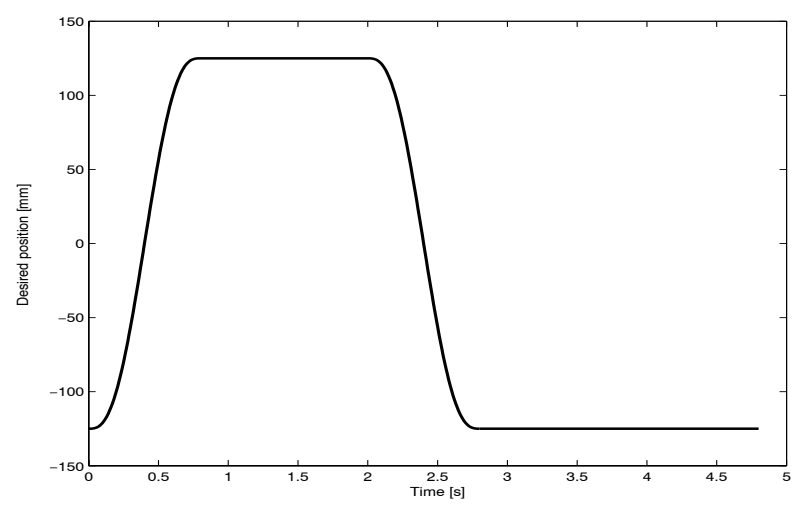

Figure 2: Desired position trajectory $(\mathrm{mm})$ versus time $(\mathrm{s})$.

\section{A second order sliding mode controller}

It is well known that the standard sliding mode features are high accuracy and robustness with respect to various internal and external disturbances. The basic idea is to force the state via discontinuous feedback to move on a prescribed manifold called the sliding manifold $\mathcal{S}^{1}=\{x \in \mathcal{X} \mid s(x, t)=0\}$ with $\mathcal{X} \subset \mathbb{R}^{n}$ so that the corresponding zero dynamics satisfy a suitable dynamical behaviour [15]. A specific problem involved by this technique is the chattering effect. Some authors $[26,29]$ relate the chattering behaviour to the discontinuity of the sign function on the sliding variable. To overcome this problem, they suggest to replace the sign function in a small vicinity of the surface by a smooth approximation; that implies a small deterioration of accuracy and robustness. A new approach called "high-order sliding mode" has been proposed [1, 19]. In this technique, instead of influencing the first sliding variable derivative, the sign function acts on its higher time derivative. Let $s(x, t)(x \in \mathcal{X})$ the sliding variable, with a relative degree equal to $r$ (i.e. the control appears in the $r^{t h}$ time-derivative of $\left.s(x, t)\right)$. In the case of the $r^{t h}$ order sliding mode, the idea is to keep the following set of constraint conditions $s(x, t)=\dot{s}(x, t)=\cdots=s^{(r-2)}(x, t)=$ $s^{(r-1)}(x, t)=0$, where $r \in I N$. In this configuration, the control $u$ acts directly on $s^{(r)}(x, t)$ but the total time derivatives $s^{(r-1)}(x, t), s^{(r-2)}(x, t), \cdots, \dot{s}(x, t), s(x, t)$ are regular continuous functions defined on the state space.

\subsection{Second order sliding mode}

Without loss of generality, consider a single-input nonlinear system

$$
\begin{aligned}
& \dot{x}=f(x)+g(x) u \\
& y=s(x, t)
\end{aligned}
$$


with $x \in \mathcal{X} \subset \mathbb{R}^{n}$ the state variable and $u \in \mathcal{U} \subset \mathbb{R}$ the input, such that $\mathcal{X}=\left\{x \in \mathbb{R}^{n}|| x_{i} \mid \leq x_{\text {MAX }}, 1 \leq i \leq n\right\}$ and $\mathcal{U}=\left\{x \in \mathbb{R}|| u \mid \leq u_{M A X}\right\} . s(x, t)$ is the output function, called sliding variable. $f, g$ and $s$ are smooth uncertain functions. Suppose that the control objective is to force $s(x, t)$ to zero. By differentiating twice $s$, under the assumption that system (5) has relative degree versus $s$ equal to 2, it leads to the following relationship

$$
\begin{aligned}
& \dot{s}=\frac{\partial s}{\partial t}+\frac{\partial}{\partial x}[s][f(x)+g(x) u] \\
& \ddot{s}=\frac{\partial^{2} s}{\partial t^{2}}+\frac{\partial}{\partial x}[\dot{s}][f(x)+g(x) u] .
\end{aligned}
$$

Definition 1 [2] Given the sliding variable $s(x, t)$, its "second order sliding manifold" is defined as

$$
\mathcal{S}=\{x \in \mathcal{X} \mid s(x, t)=\dot{s}(x, t)=0\}
$$

Definition 2 Consider the not-empty second order sliding set (7), and assume that it is locally an integral set in the Filippov sense, i.e. it consists of Filippov's trajectories of the discontinuous dynamics system [11]. The corresponding behavior of system (5) satisfying (7) is called "second order sliding mode" with respect to the sliding variable $s(x, t)$.

Definition 2 means that system (5) satisfies a second order sliding mode with respect to $s(x, t)$ if its state trajectories lie on the intersection of the two manifolds $s(x, t)=0$ and $\dot{s}(x, t)=0$ in the state space. In order to state a rigorous control problem, the following conditions are assumed:

- H1 $u$ is bounded and discontinuous. Furthermore the solution of (5) admits solution in the Filippov sense on the 2-sliding manifold $\mathcal{S}$ for all $t$

- $\mathrm{H} 2\|f(x)\|_{2}$ and $\|g(x)\|_{2}$ are bounded and $\frac{\partial}{\partial u} \ddot{s}(x, t)>0$.

Let $a=\frac{\partial^{2} s}{\partial t^{2}}+\frac{\partial}{\partial x}[\dot{s}] f(x), \quad$ and $\quad b=\frac{\partial}{\partial x}[\dot{s}] g(x)$. Under $\mathrm{H} 1-\mathrm{H} 2$, there exist positive constant values $C, K_{m}$ and $K_{M}$ so that, $\forall u \in \mathcal{U}$ and $\forall x \in \mathcal{X}$,

$$
0<K_{m}<b(x, u, t)<K_{M}, \quad|a(x, u, t)| \leq C
$$

Consider local coordinates $\left[\zeta_{1} \zeta_{2}\right]^{T}=\left[\begin{array}{l}s \\ \dot{s}\end{array}\right]^{T}$. Then, on the basis of the previous definitions and conditions, the second order sliding mode problem may be expressed in terms of the finite time stabilization problem for the following uncertain second order system [19]

$$
\begin{aligned}
& \dot{\zeta}_{1}=\zeta_{2} \\
& \dot{\zeta}_{2}=a(x, u, t)+b(x, u, t) u
\end{aligned}
$$

where $\zeta_{2}$ may be unmeasurable but with a known sign. There exist several algorithms able to ensure the finite time stabilization of the system (9) towards the origin [1, 12, 19]. Among them, the so-called "Twisting algorithm" is based on an adequate commutation of the control between two different values so that the trajectories in the phase plan of (9) execute an infinite number of rotations while converging in finite time to the origin. This algorithm is defined by the following control law $[12,19]$

$$
u= \begin{cases}-\alpha_{m} \operatorname{sign}\left(\zeta_{1}\right) & \text { if } \zeta_{1} \zeta_{2} \leq 0 \\ -\alpha_{M} \operatorname{sign}\left(\zeta_{1}\right) & \text { if } \zeta_{1} \zeta_{2}>0\end{cases}
$$

with the sufficient conditions which ensure the finite time convergence to the sliding manifold

$$
\begin{aligned}
& 0<\alpha_{m}<\alpha_{M}, \quad \alpha_{m}>\frac{C}{K_{m}} \\
& K_{m} \alpha_{M}-C>K_{M} \alpha_{m}+C .
\end{aligned}
$$

\subsection{Sliding variable and control design}

To track the load position given by Figure 2, a sliding variable is proposed, so that the error dynamics follows a desired first order dynamic. Denoting $x_{4_{r e f}}(t)$ the desired trajectory, one gets

$$
s=x_{3}-\dot{x}_{4_{r e f}}+\lambda\left(x_{4}-x_{4_{r e f}}\right)
$$

where $\lambda$ is a positive parameter such that $P(z)=\dot{z}+\lambda z$ is Hurwitz polynomial. Note that the relative degree of $s$ equals 2. Consider the second time derivative of $s$

$$
\ddot{s}=A+B u
$$

where

$$
\begin{aligned}
A= & \frac{k_{7}}{V_{P}\left(x_{4}\right)}\left[k_{1}+k_{3} x_{1} x_{3}\right]+\frac{k_{8}}{V_{N}\left(x_{4}\right)}\left[k_{4}+k_{6} x_{2} x_{3}\right] \\
& +k_{9}\left[k_{7} x_{1}+k_{8} x_{2}+k_{9} x_{3}+F\right]+\dot{F}-x_{4 r e f}^{(3)} \\
& +\lambda\left[k_{7} x_{1}+k_{8} x_{2}+k_{9} x_{3}+F-\ddot{x}_{4 r e f}\right] \\
=: & A_{0}+\delta A \\
B= & \frac{k_{7} k_{2}}{V_{P}\left(x_{4}\right)}+\frac{k_{8} k_{5}}{V_{N}\left(x_{4}\right)} \\
= & B_{0}+\delta B
\end{aligned}
$$

$A_{0}$ and $B_{0}$ are the known nominal expressions whereas the expressions $\delta A$ and $\delta B$ contain all the uncertainties due to parameters variations and term $F$. The final controller is composed by a linearizing controller coupled to a second order sliding mode one (see Figure 3 and Equation (15)). The validity of the control law depends on the stability of the unobservable subsystem, which is one-dimensional. It is very difficult to obtain results about the global stability of the zero dynamics, but, in the physical domain, the local stability has been proved [6]. Using the static feedback ${ }^{2}$

$$
u=B_{0}^{-1}\left[-A_{0}+v\right]
$$

where $v$ is the new control vector, one gets

$$
\begin{aligned}
\ddot{s} & =\left[\delta A-\frac{\delta B}{B_{0}} A_{0}\right]+\left[1+\frac{\delta B}{B_{0}}\right] v \\
& =: \bar{A}+\bar{B} v
\end{aligned}
$$

\footnotetext{
${ }^{2}$ The scalar $B_{0}$ never equals 0 ; then, the control input $u$ is always defined.
} 


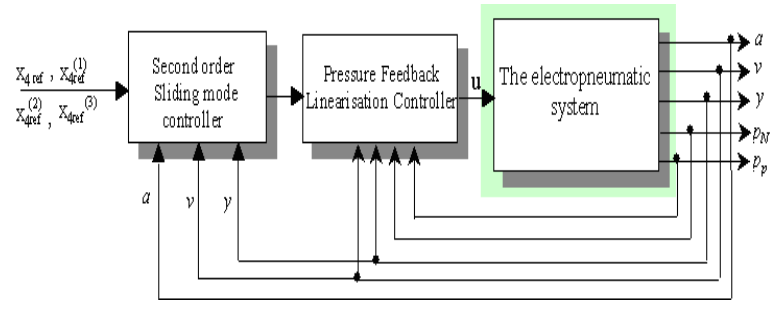

Figure 3: Global structure of the robust controller.

In fact, the term $-B_{0}^{-1} A_{0}$ of (15), which is the so-called equivalent control in the sliding mode context [29], is not able to cancel all the nonlinearities. Then, uncertainties can act on the motion equations in a nonlinear form. Since $x_{1}, x_{2}, x_{3}, x_{4}$, $k_{i}, \delta k_{i}, F$ and $\dot{F}$ are bounded, there exist positives constants $C, K_{m}$, and $K_{M}$ so that

$$
\begin{aligned}
& |\bar{A}|<C \\
& 0<K_{m}<\bar{B}<K_{M}
\end{aligned}
$$

Then, one can apply the second order algorithm previously presented

$$
v= \begin{cases}-\alpha_{m} \operatorname{sign}(s) & \text { if } s \dot{s} \leq 0 \\ -\alpha_{M} \operatorname{sign}(s) & \text { if } s \dot{s}>0\end{cases}
$$

with

$$
\begin{aligned}
& 0<\alpha_{m}<\alpha_{M}, \quad \alpha_{m}>\frac{C}{K_{m}} \\
& K_{m} \alpha_{M}-C>K_{M} \alpha_{m}+C .
\end{aligned}
$$

Finally, a sliding mode occurs on $\mathcal{S}$ leading to desired tracking property for the position.

\section{Implementation results of the second order sliding mode controller}

The control law is implemented using a dSpace DS1104 controller board with a dedicated digital signal processor with a $4 m s$ sample time. Two pressure sensors are fixed in each chamber. The sensed signals were run through the signal conditioning unit before being read by the 16 bits analog/digital converter. The pressures $p_{N}$ and $p_{P}$ are such that $x_{\text {min }}=1$ bar and $x_{M A X}=7$ bar. The maximum/minimum value of the load position equals $x_{4 \min / 4 M A X}= \pm 250 \mathrm{~mm}$. The control input is such that $u_{M A X}=10 \mathrm{~V}$

The objective consists in minimizing the position tracking error in presence of model uncertainties and load variations. The gains $\alpha_{m}$ and $\alpha_{M}$ have been tuned such that condition (18) is satisfied : $\alpha_{m}=200$ and $\alpha_{M}=7000$. The real $\lambda$ is fixed at $\lambda=15 s^{-1}$. Some experiment results are provided here to demonstrate the robustness of the second order sliding mode controller. Firstly, the total load mass equals $27 \mathrm{~kg}$. Figure 4 displays the tracking position error with desired position described in Section 2.5. The maximum position tracking error is about $2.12 \mathrm{~mm}$ which is better than with classical nonlinear control $[8]^{3}$ : this error represents less than $1 \%$ of the total

\footnotetext{
${ }^{3}$ The control laws in [8] have been implemented on the same experimental set-up, in the same conditions.
}

displacement magnitude. In steady state, the position error is about $86 \mu \mathrm{m}$, which is better than with classical linear control law (PI) [14]. Figure 5 displays the control input which is not

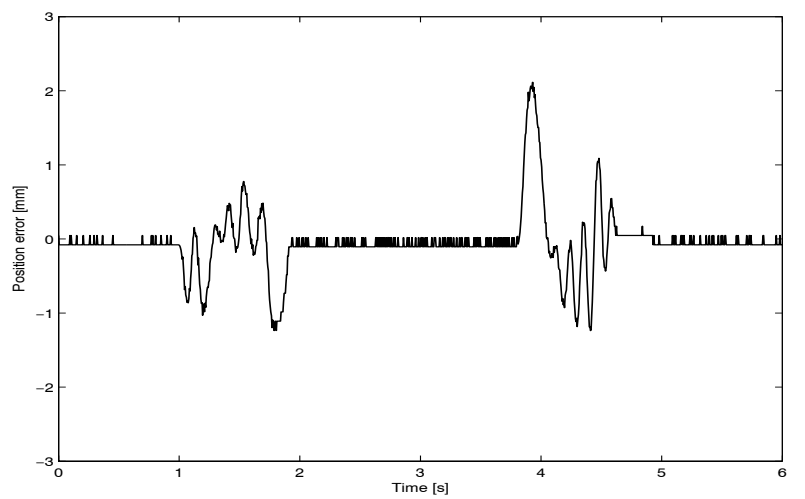

Figure 4: Tracking position error $(\mathrm{mm})$ versus time $(\mathrm{s})$.

affected by the chattering effect. Even if the signal exited the spool valve during the dynamic stage, no audible noise can be heard $^{4}$, which was not the case with first order sliding [5]. From these experiment results, good tracking responses are obtained for the position owing to the robust control characteristics of the controller. These two curves are obtained without a good mass flow rate model and in presence of important frictions variations. For the last experimentation, the total load mass

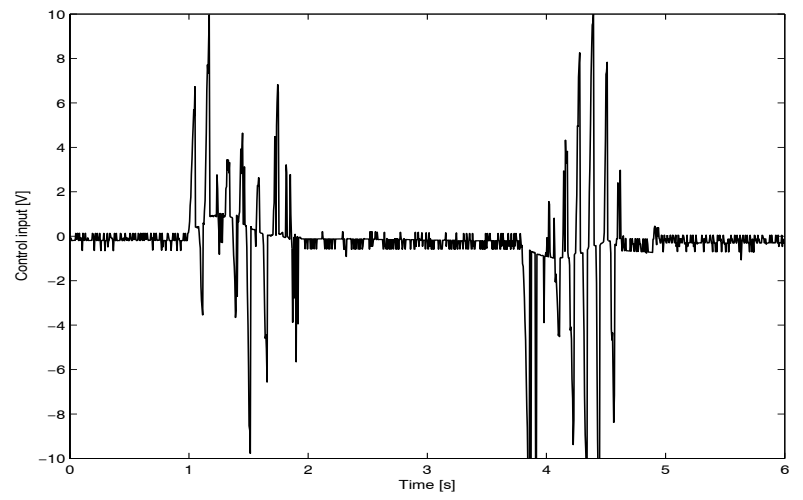

Figure 5: Control input $(V)$ versus time $(s)$.

is decreased until $17 \mathrm{~kg}$. The presented results are obtained without changing the control gains value. The robust control characteristics of this controller versus the load mass variation can be observed in Figure 6. The maximum position tracking error is about $1.81 \mathrm{~mm}$. In steady state, the position error is about $92 \mu \mathrm{m}$. The required performances are achieved.

\section{Conclusion}

The paper has proposed a second order sliding mode controller for an electropneumatic actuator. The controller based on the

${ }^{4}$ The noise is due to a very high frequency displacement of the servodistributor mobile part, which can induce a faster wear. 


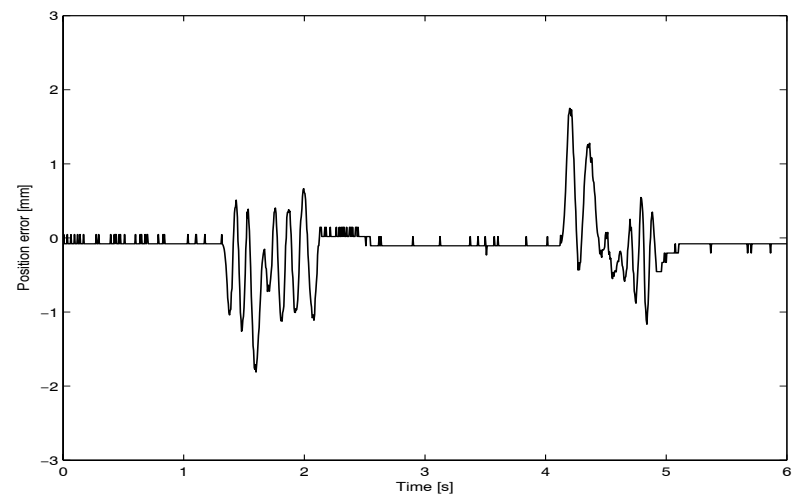

Figure 6: Tracking position error $(\mathrm{mm})$ versus time $(\mathrm{s})$.

twisting algorithm has been tuned so that its convergence is ensured in spite of parameters uncertainties and perturbation. Experimental results show that the trajectory tracking is done with a very good accuracy. The results have been compared to previous ones and appear more precise and robust versus uncertainties and load variations.

\section{References}

[1] G. Bartolini, A. Ferrara, and E. Usai, "Chattering avoidance by second-order sliding mode control", IEEE Trans. Automat. Control, vol. 43, no.2, pp.241-246, (1998).

[2] G. Bartolini, A. Ferrara, A. Pisano, and E. Usai, "On the convergence of 2-sliding algorithm for non-linear uncertain systems", International Journal of Control, vol. 74, no.7, pp.718-731, (2001).

[3] M. Belgharbi, D. Thomasset, S. Scavarda, and S. Sesmat, "Analytical model of the flow stage of a pneumatic servodistributor for simulation and nonlinear control", Proc. Sixth Scandinavian International Conference on Fluid Power SICFP'99, Tampere, Finland, pp.847-860, (1999).

[4] M. Bouri, D. Thomasset, and S. Scavarda, "Integral sliding mode controller of a rotational servodrive", Proc. $3^{\text {rd }}$ JHPS, Tokyo, Japan, pp.145-150, (1996).

[5] M. Bouri, D. Thomasset, "Sliding control of an electropneumatic actuator using an integral switching surface", IEEE Trans. Control Syst. Technology, vol.2, no.2, pp.368-375, (2001).

[6] X. Brun, M. Belgharbi, S. Sesmat, D. Thomasset, and S. Scavarda, "Control of an electropneumatic actuator, comparison between some linear and nonlinear control laws", Journal of Systems and Control Engineering, vol.213, no.I5, pp.387-406, (1999).

[7] X. Brun, S. Sesmat, D. Thomasset, and S. Scavarda, "A comparative study between two control laws of an electopneumatic actuator", Proc. European Control Conference ECC'99, Karlsruhe, Germany, (1999).
[8] X. Brun, D. Thomasset, "Choice of control law in electropneumatics. Expertise using an industrial benchmark and some new trends", Proc. Conference on Decision and Control CDC'O0, Sydney, Australia, (2000).

[9] X. Brun, D. Thomasset, and E. Bideaux, "Influence of the process design on the control strategy: application in electropneumatic field", Control Engineering Practice, vol.10, no.7, pp.727-735, (2002).

[10] K.A. Edge, "The control of fluid power systems - responding to the challenge", Journal of Systems and Control Engineering, vol.211, no.I2, pp.91-110, (1997).

[11] A.F. Filippov, Differential Equations with Discontinuous Right-Hand Side, Kluwer, Dordrecht, the Netherlands, (1988).

[12] S.V. Emelyanove, S.K. Korovin, and A. Levant,"Higherorder sliding modes in control systems", Differential Equations, vol.29, no.11 pp.1627-1647,(1993).

[13] K. Furuta, "Sliding mode control of discrete system", Systems and Control Letters, vol.14, pp.145-152, (1990).

[14] K. Hamiti, A. Voda-Besançon, and H. Roux-Buisson, "Position control of a pneumatic actuator under the influence of stiction", Control Engineering Practice, vol.4, no.8, pp.1079-1088, (1996).

[15] A. Isidori, Nonlinear Control Systems, Spriger-Verlag, New York, (1989).

[16] T. Kimura, S. Hara, T. Fujita, and T. Kagawa, "Feedback linearization for pneumatic actuator systems with static friction", Control Engineering Practice, vol.5, no.10, pp.1385-1394, (1997).

[17] S. Laghrouche, F. Plestan, A. Glumineau, and R. Boisliveau, "Robust second order sliding mode control for a permanent magnet synchronous motor", Proc. American Control Conference ACC'03, Denver, Colorado, 4-6 juin, 2003.

[18] S. Laghrouche, F. Plestan, and A. Glumineau, "Higher order sliding mode control based on optimal linear quadratic control", Proc. 7th European Control Conference ECC'03, Cambridge, Angleterre, 1-4 septembre, 2003.

[19] A. Levant, "Sliding order and sliding accuracy in sliding mode control", International Journal of Control, vol.58, no.6, pp.1247-1263, (1993).

[20] D. Mac Cloy, "Discharge characteristics of servo valve orifices", Proc. Fluid Power International Conference, Olympia, Greece, pp.43-50, (1968).

[21] S. Ming-Chang, and T. Shy-I, "Identification and position control of a servo pneumatic cylinder", Control Engineering Practice, vol.3, no.9, pp.1285-1290, (1995). 
[22] A.K. Paul, J.K. Mishra, M.G. Radke, "Reduced order sliding mode control for pneumatic actuator", IEEE Trans. Control Syst. Technology, vol.2, no.3, (1994).

[23] E. Richard, and S. Scavarda, "Comparison between linear and lonlinear control of an electropneumatic servodrive", J. of Dynamic Systems, Measurement, and Control, vol.118, pp.245-252, (1996).

[24] S. Sesmat, and S. Scavarda, "Static characteristics of a three way servovalve", Proc. 12th Conference on Fluid Power Technology, Aachen, Allemagne, pp.643652, (1996).

[25] J.L. Shearer, "Study of pneumatic processes in the continuous control of motion with compressed air", Trans. Am. Soc. Mech. Eng., vol.78, pp.233-249, (1956).

[26] J.J. Slotine, "Sliding mode controller designe for nonlinear systems", International Journal of Control, vol.40, no.2, pp.421-434, (1984).

[27] L.R. Tokashiki Kishimoto, Dynamic characteristics of pneumatic cylinder systems, Ph.D. Thesis, Tokyo Institute of Technology, 133p, (1999).

[28] V.I. Utkin, "Variable structure systems with sliding modes", IEEE Trans. Automat. Control, vol.26, no.2, pp. 212-222, (1977).

[29] V.I. Utkin, Sliding Mode in Control and optimization, Springer-Verlag, Berlin, (1992).

[30] L. Yang, J.H. Lilly, "Sliding mode tracking for pneumatic muscle actuators in bicep/tricep pair configuration", Proc. American Control Conference ACC'03, Denver, Colorado, pp.4669-4672, (2003).

[31] "Servo pneumatics finding its niche, bit by bit", Control Engineering Europe, vol.4, no.1, pp.32-33, (2003). 